\title{
USO OFF-LABEL DE MEDICAMENTOS PARA TRATAMENTO DE DOENÇAS CARDIOVASCULARES EM CRIANÇAS DE 0 A 12 ANOS.
}

\section{Caio Luiz Lopes', Gabrielle Marques de Fazio Cristovão', Larissa Valeska do Nascimento Rodrigues', Rafael Matheus Taveira', Luma Arakelian Penna', Marcela Henrique de Oliveira Cruz', Leandro Giorgetti ${ }^{2, A}$}

1'Discentes do Curso de Farmácia da Universidade Anhembi Morumbi - São Paulo - Brasi

Docente do Curso de Farmácia da Universidade Anhembi Morumbi - São Paulo - Brasil

\section{RESUMO}

Devido à escassez de dados, o uso de medicamentos off-label se tornou uma opção recorrente de tratamento para o público infantil, principalmente na área das doenças cardiovasculares. Objetivo: Avaliar a frequência de prescrição de medicamentos off-label para crianças e identificar para quais doenças foi necessária a utilização desses medicamentos, bem como analisar as principais causas para esse uso. Fonte de dados: Artigos acadêmicos provenientes de diversas bases de dados, no período de novembro de 2020 a outubro de 2021. Síntese de dados: A predominância das prescrições fora da bula ocorreu entre as crianças de 1 a 2 anos de idade que tenham algum tipo de patologia cardíaca e que o motivo mais utilizado para a uso off-label foi relacionado ao ajuste dose e frequência. Por fim, identificamos alguns medicamentos que possuem indicação de bula e fizemos um levantamento de dados para comparar com a indicação off-label. Conclusão: Evidente a necessidade de pesquisas que priorizem o conhecimento farmacocinético e farmacodinâmico na população pediátrica, além do reforço nas instruções aos profissionais de saúde, com a finalidade de racionalizar o tratamento e buscar evidência científica que suporte o uso off-label de medicamentos.

Palavras-chave: Uso off-label, pediatria, cardiopatias, farmacoterapia, cardiologia.

\section{ABSTRACT}

Due to the scarcity of data, the use of off-label medications has become a recurrent treatment option for children, especially in the area of cardiovascular diseases. Objective: To assess the frequency of off-label drug prescription for children and identify which diseases the use of these drugs was necessary for, as well as analyze the main causes for this use. Data source: Academic articles from several databases, from November 2020 to October 2021. Data synthesis: Predominance of package insert prescriptions occurred among children aged 1 to 2 years who had some type of cardiac pathology and that the most used reason for off-label 
use was related to dose and frequency adjustment. Finally, it identifies some drugs that have indications on the package insert and a data collection to compare with an off-label indication. Conclusion: The need for research that prioritizes pharmacokinetic and pharmacodynamic knowledge in the pediatric population is evident, in addition to strengthening the instructions to health professionals, in order to rationalize the treatment and seek scientific evidence to support the off-label use of medicines.

Key words: Off- label use, pediatrics, heart disease, drug therapy, cardiology

\section{INTRODUÇÃO}

No Brasil, o processo de desenvolvimento e aprovação de medicamentos dependem de uma série de estudos, tais como os pré-clínicos, in vitro e in vivo, que tem como foco o desenvolvimento da molécula, elucidação do mecanismo de ação, dosagem e segurança. Após esse processo, caso não ocorram intercorrências, o ensaio clínico prossegue em humanos de fase I, II e III, em que são observados pontos relevantes como toxicidades, farmacocinética, segurança e eficácia, o que garante qualidade do medicamento. De acordo com o Instituto Nacional de Câncer (INCA), em um levantamento feito a respeito da quantidade de moléculas utilizadas nos estudos, o processo de desenvolvimento e aprovação pode levar mais de 10 anos e, de aproximadamente 1000 moléculas, apenas uma terá sua segurança e eficácia comprovada e aprovada. $[1,2]$

$O$ registro definitivo de um fármaco é regido pela Agência Nacional de Vigilância Sanitária (ANVISA), por meio da Lei $n^{\circ}$ 6.360, de 23 de setembro de 1976.[3] A ANVISA é a organização que fica responsável por monitorar toda a fase de estudos e ao final do processo conceder o registro do medicamento a empresa detentora, afirmando que ele é seguro e eficaz para a finalidade terapêutica descrita em bula. Por outro lado, os medicamentos aprovados pela ANVISA e outras agências continuam sendo estudados para outras finalidades terapêuticas, inclusive administrados para pacientes com doenças que não são o alvo da patologia de indicação. Isso ocorre, pois em muitas situações existem dificuldades de encontrar a forma farmacêutica, dosagem, via de administração ou medicamento exclusivo.[4]

Nesse sentido, todo medicamento alopático com finalidade terapêutica definida que é utilizado sem indicação da bula se denomina off-label. O termo é designado a tudo que tiver seu uso irrestrito ao da bula, ou seja, para qual foi inicialmente criado. Essa prática clínica é amplamente aplicada em todo mundo sofrendo algumas alterações em diferentes países.[5]

Segundo alguns autores, existe uma diferença significativa entre o uso off-label dos medicamentos e do uso experimental em pesquisas, pois o uso experimental utiliza novas drogas ou drogas já comercializadas para fazer pesquisa em cima delas, diferentemente do off-label, que utiliza um medicamento já existente com uma finalidade descrita, para ser usado em uma outra situação onde, em muitos casos, não existem estudos e pesquisas científicas que comprovem sua verdadeira eficácia. Isso serve para alertar muitos profissionais do setor da saúde para que nenhum uso em paciente tenha sua segurança comprometida. $\mathrm{O}$ uso off-label é incompatível com o uso racional de medicamentos (URM) promovido pela OMS em 1985, que tem como fundamento a utilização de medicamentos adequados ao tratamento do paciente, em doses corretas, período adequado e com o menor custo possível, atendendo às suas necessidades. [6,7]

Conforme Diel et al. (2020) o Brasil é um dos países com maior taxa de utilização de prescrição pediátrica de modo off-label. Assim como nos EUA, possui uma predominância na utilização de prescrições off-label devido à idade e indicação, de modo que $62 \%$ foram para pacientes de 0 a 17 anos. Na Holanda, 22,7\% das prescrições realizadas para pacientes nessa mesma faixa etária foram consideradas off-label, e na Alemanha, $40,2 \%$ sendo que suas maiores frequências foram devidas às subdosagens, sobredosagens, indicação e idade. [8]

Dado isto, conseguimos analisar a grande quantidade de prescrição off-label para o público infantil, de forma que é possível sugerir a falta de disponibilidade de medicamentos registrados com a formulação adequada para a faixa etária, e isto é dado a razões multifatoriais, como escassez de evidências científicas, diferenciação biológica no período de 0 a 12 anos (neonatal, criança, pré-adolescente e adolescente), questões éticas e razões econômicas que desencorajam o investimento das empresas farmacêuticas.[9]

Diante disto nosso objetivo é realizar uma revisão bibliográfica voltada ao uso de medicamentos off-label na população pediátrica, de acordo com as principais cardiopatias que as acometem, e ainda descrever os medicamentos mais utilizados para os pacientes pertencentes a essa faixa etária.

\section{MATERIAIS E MÉTODOS}

O presente trabalho trata-se de uma revisão bibliográfica, que tem como objetivo identificar e avaliar os principais usos de medicamentos off-label na pediatria cardiológica. Além disso, cita os riscos e benefícios desse tipo de prática, que vem se desenvolvendo e ganhando espaço como alternativas de tratamento, abrindo assim um debate sobre o assunto por ser um tema em constante discussão pelos profissionais da saúde.

Para a execução deste trabalho, foram realizadas buscas em bases de dados - PubMed, Google Acadêmico, SciELO. Ao finalizar as pesquisas em cada base, as referências duplicadas foram excluídas. Os critérios utilizados para as pesquisas foram, data de publicação com enfoque entre os anos de 2016 a 2021, 
relevância do artigo, preferência para textos em inglês e português, e ainda foram delimitadas palavras chaves para encontrar artigos que correspondessem melhor ao tema. Entre livros, artigos, periódicos e legislações, foram analisados cerca de 70 materiais.

Em conjunto com as bases de dados padrão, foram desenvolvidas bases de dados complementares como mapa mental, planilhas, cronogramas de andamento e entrega do trabalho. De modo que proporcionou uma melhor organização e otimização do tempo.

\section{RESULTADOS E DISCUSSÃO}

\section{HISTÓRICO DO USO OFF-LABEL DE MEDICAMENTOS}

Historicamente, o off-label é uma conduta existente mesmo antes do século $\mathrm{XX}$. Os pioneiros na área da saúde buscavam outros métodos para solucionar os casos de doenças apresentadas pela população da época, transformando a descoberta científica em alternativa de tratamento.[10]

Um dos primeiros relatos do uso off-label aconteceu em 1930, quando o médico psiquiatra e neurologista William Bleckwenn utilizou o Amital de Sódio, medicamento classificado como anestésico e ansiolítico para tratamento de pacientes com síndrome comportamental conhecida como catatonia. [10] Em meados de 1940, um novo estudo foi iniciado com o sulfato de quinina, pois antigamente era usado para tratamento de cãibras musculares recorrentes. Entretanto em 2006 foi divulgado pelo Registro Federal da Food and Drug Administration (FDA) nos EUA que sulfato de quinino não é aprovado para este tipo de conduta devido à grande quantidade de efeitos adversos, sendo assim seu uso se tornou exclusivo no tratamento de malária por Plasmodium falciparum. [11,12, 13]

Segundo Tukayo et al. (2020), é possível notar que a prática de prescrição off-label passou por várias décadas, se tornando cada vez mais frequente à medida que a sociedade evoluiu juntamente com a ciência e tecnologia. A partir dos dados levantados na pesquisa, dos 1.961 medicamentos prescritos $71,5 \%$ são offlabel. Com base nesse resultado, é possível verificar a recorrência da utilização da prescrição off-label com o passar dos anos. [14]

\section{PAPEL DAS AGÊNCIAS REGULADORAS}

As agências reguladoras, como a ANVISA, detêm a responsabilidade da aprovação dos medicamentos comercializados, o que garante o controle da segurança, eficácia e qualidade dos produtos através de diversas regulamentações e normas. Este processo é efetuado através da verificação da legitimidade e da compatibilidade dos dados fornecidos pelo fabricante do medicamento. [15, 16]

Em casos de prescrição off-label, que não tem sua indicação aprovada pelas agências regulatórias, pode ocorrer aumento dos eventos adversos, pois não há um estudo específico e com verificação adequada para garantir a segurança do paciente.[17] Com isto, esse tipo de tratamento deve ser utilizado em casos individuais com o respaldo de dados científicos, para beneficiar o paciente que não possui um tratamento com produtos registrados para tal indicação. $[18,19]$

Com o objetivo de regulamentar os prescritores, o Conselho Federal de Medicina emitiu o Parecer $n^{\circ}$ 13/2004 e 2/2016 e as Resoluções $n^{\circ} 2.222 / 2018$ e 2.226/2019, que descreve a concessão de prescrever medicamentos fora dos termos apresentados em bula (off-label) ou de medicamentos não licenciados no país da prescrição, desde que tenha o consentimento do paciente, o registro das motivações de tal conduta e a ciência da responsabilidade assumida que poderá condicionar em penalidade. [20, 21, 22]

Dessa forma, à espera da atualização de regulamentações e a ausência de pesquisas realizadas pelos laboratórios para a comprovação de novas indicações para as agências reguladoras estimularam as prescrições off-label entre vários tipos de pacientes, dentre eles, os pediátricos. [15, 23, 24]

\section{USO OFF-LABEL EM PEDIATRIA}

A área da pediatria vem sendo um dos maiores retentores de prescrições off-label de acordo com Silveira (2019), mais de 50\% dos medicamentos utilizados pelo público infantil são deste tipo de prescrição, onde em alguns países a porcentagem pode até ser superior a esta apresentada. [8, 25, 26]

No Brasil nos anos de 2013 a 2014, os autores Diel et. al realizaram um estudo populacional que indica o percentual de prescrições off-label de polifarmácia de acordo com a idade das 7.528 crianças participantes da pesquisa nacional. 8 Nesse caso, é possível observar que a maior prevalência é para os indivíduos entre 1 a 2 anos de idade (Figura 1).

Figura 1: Quantidade de crianças em uso de polifarmácia off - label de acordo com idade.

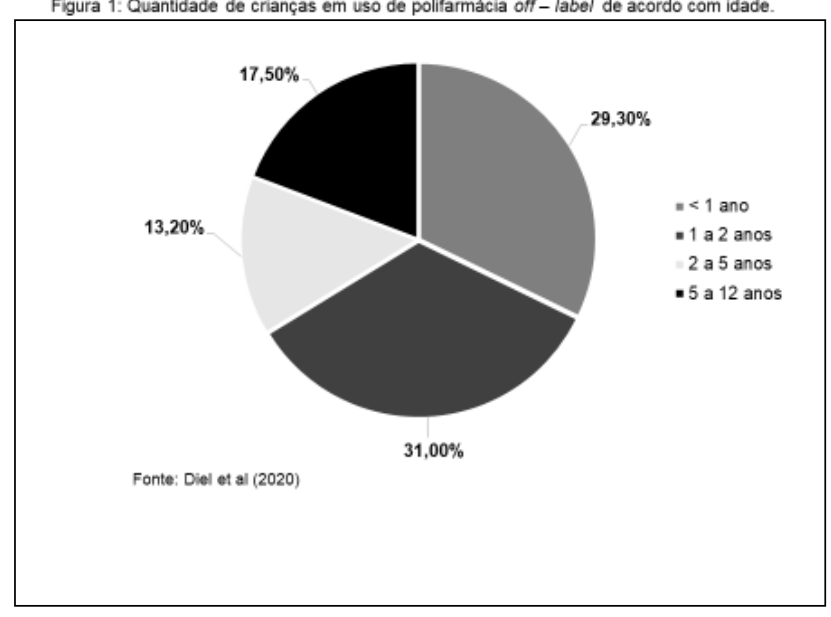

Fonte: Adaptado de Diel, 2020.

As causas para a prática da terapia off-label na população pediátrica são a falta de formulações e formas farmacêuticas específicas disponíveis no mercado, carência de evidências sobre a eficácia e segurança comprovadas em crianças e indisponibilidade 
de tratamento terapêutico adequado a esse público. $[8,27,28]$ Essa problemática se dá pela falta de pesquisa clínica utilizando crianças, principalmente a longo prazo, devido a questões éticas e de riscos a esse grupo de pacientes. [29] Em 2017, o Ministério da Saúde (MS) publicou um documento que consta apenas $8 \%$ do número total de estudos sobre medicamentos era voltado à participação de crianças, tanto no cenário brasileiro quanto global. [30]

Por esses motivos, a prescrição de medicamentos em pediatria acaba seguindo os mesmos critérios adotados para adultos, mesmo sendo comprovado que até o segundo ano de vida o sistema enzimático de metabolização das drogas e principalmente a função renal são diferentes em comparação com indivíduos adultos. Além disso, as características farmacocinéticas e farmacodinâmicas modificam-se ao longo do tempo e do crescimento da criança, em virtude da imaturidade hepática, variações do $\mathrm{pH}$ estomacal e eventuais deficiências enzimáticas próprias da idade, o que implica diretamente na terapêutica. [31]

\section{USO OFF-LABEL PARA PACIENTES PEDIÁTRICOS COM DOENÇAS CARDIOVASCULARES}

Conforme apresentado por Selig (2020), cerca de 30.000 recém-nascidos podem ser portadores de doenças cardíacas. [32] Essas cardiopatias atingem não apenas o coração como também artérias, vasos, sistemas corporais e demais órgãos de uma forma específica ou generalizada no corpo humano, resultando assim, em tratamento farmacológico que, dependendo do quadro clínico, pode gerar uma internação ou intervenção cirúrgica. [33, 34]

As doenças cardíacas na população infantil podem apresentar sinais e sintomas muitas vezes imperceptíveis. Sendo que o diagnóstico e a intervenção podem ocorrer durante a gestação, ou após o nascimento do indivíduo. Para pacientes na fase da infância o diagnóstico somente é realizado quando é verificado por um especialista. [35]

Segundo um estudo feito por Gonçalves e Heineck (2015), foi realizada a análise das prescrições de 326 pacientes, dentro do período de agosto a dezembro de 2012 no Rio Grande do Sul Brasil, concluíram que $31,7 \%$ dos medicamentos prescritos são off-label; dentre eles as categorias mais recorrentes foram: dose com $38,8 \%$, seguida de faixa etária $31,5 \%$ e de frequência de administração 29,3\%. [36]

Em contribuição com os demais estudos feitos acima, Costa et.al (2018) realizaram um levantamento dos principais tipos de prescrições off-label evidenciado na figura 2 com o total de 17.421 prescrições pediátricas. [37]

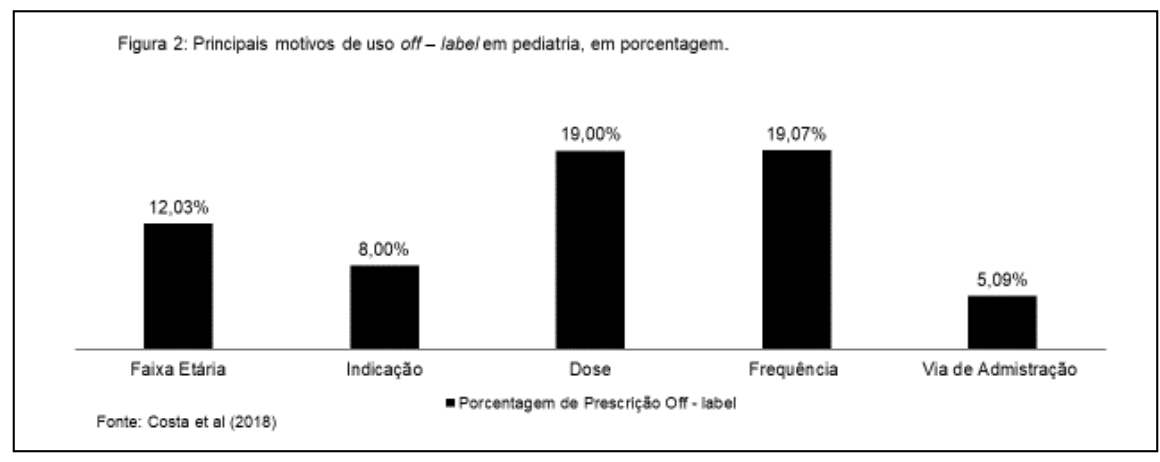

Fonte: Adaptado de Costa, 2018

Com base na Figura 2, foi desenvolvida a tabela abaixo que aponta as principais utilizações off-label classificadas quanto a dosagem, faixa etária e indicação terapêutica, os medicamentos mais utilizados e para quais cardiopatias foram aplicadas. [38]

Tabela 1 - Fármacos indicados de acordo com a categoria off-label, para aplicação em determinadas cardiopatias. [39, 40]

\begin{tabular}{|c|c|c|}
\hline Fármacos indicados & Categorias off-label & Patologia \\
\hline Amiodarona & Subdose e Indicação & Arritmia e Taquiarritmia \\
\hline Atenolol & Faixa Etária e Subdose & Taquiarritmias supraventriculares e ventriculares \\
\hline Bisoprolol & Faixa Etária & Insuficiência Cardíaca \\
\hline
\end{tabular}


LOPES, C.L. E COLABORADORES - REVISTA BRASILEIRA DE CIÊNCIAS BIOMÉDICAS - VOLUME 2

\begin{tabular}{|c|c|c|}
\hline Captopril & Faixa Etária, Subdose e Indicação & Insuficiência Cardíaca Crônica \\
\hline Carvedilol & Faixa Etária e Subdose & Insuficiência Cardíaca Congestiva \\
\hline Digoxina & Indicação & Insuficiência cardíaca crónica, arritmia e taquicardia \\
\hline Dobutamina & Subdose e Indicação & Insuficiência cardíaca \\
\hline Enalapril & Subdose & Insuficiência cardíaca \\
\hline Esmolol & Faixa Etária e Subdose & Taquicardia supraventricular e Arritmia. \\
\hline Hidroclorotiazida & Subdose & Insuficiência cardíaca \\
\hline Levosimendan & Faixa Etária, Subdose e Indicação & Cardiopatia congênita \\
\hline Lidocaína & Indicação & Taquicardia \\
\hline Metoprolol & Faixa Etária, Subdose e Indicação & Taquiarritmias e Insuficiência cardíaca \\
\hline Nitroglicerina & Faixa Etária e Subdose & Insuficiência cardíaca congestiva \\
\hline Pentoxifilina & Faixa Etária e Subdose & Doença de Kawasaki \\
\hline Peridopril & Faixa Etária & Insuficiência cardíaca congestiva \\
\hline Propanolol & Indicação & Arritmia e Taquiarritmia \\
\hline Sotalol & Faixa Etária e Subdose & Arritmias Ventriculares e Supraventriculares \\
\hline
\end{tabular}

Fonte: Adaptado de Comité de Medicamentos de la Asociacíon Española de Pediatría, 2015 e ANVISA, 2021"

De acordo com as doenças listadas na tabela 1, o uso off-label nos últimos anos se tornou uma linha de tratamento para doenças cárdicas para população pediátrica. Dentre elas, a doença de Kawasaki, considerada a segunda vasculite mais comum na pediatria afetando principalmente crianças com idade inferior a 5 anos do sexo masculino, e é caracterizado por causar inflamações (vasculite) aguda e sistêmica que atinge órgãos, tecidos e artérias de pequeno e médio calibre tendo como principais as coronárias, que quando não tratadas são responsáveis por complicações ao sistema cardíaco, se tornando fator de desenvolvimento do Aneurisma Arterial Coronariano (AAC), que segundo representa de $15 \%$ à $25 \%$ dos casos apresentados pela população pediátrica em hospitais. [41, 42, 43, 44, 45]

Um dos medicamentos utilizados para doença de Kawasaki é a Pentoxifilina, sua indicação é para alterações tróficas, diabética, inflamatória e gangrena. Em casos específicos, como o off-label, a Pentoxifilina é utilizada em tratamentos pediátricos pelo seu efeito anti-inflamatório. Sua categoria off-label conforme a tabela é de faixa etária e subdose. [39 46]
Por outro lado, a insuficiência cardíaca ocorre quando o metabolismo corporal é maior do que o bombeamento sanguíneo e com isso, ao decorrer do tempo, serão ativadas diversas atividades intrínsecas que tem a finalidade de compensar essa insuficiência. [46] Na maioria dos casos, a contratilidade do miocárdio é afetada, causando isquemia. A dimensão dessa patologia na população pediátrica, até o momento, é desconhecida justamente por não ser padronizado nenhum formulário para identificação nessa população, entretanto, o único dado que foi possível analisar é que cerca de $15 \%$ - $25 \%$ de pacientes que nasceram com alterações estruturais cardíacas irão desenvolver insuficiência cardíaca. [48, 49, 50]

A Nitroglicerina é um dos medicamentos de escolha para IC, utilizada como off-label de indicação, já que é utilizada para insuficiência cardíaca crônica, urgência cardíaca, angina secundária devido ao uso de cocaína entre outros. A justificativa para esse uso pode estar relacionada com o mecanismo de ação que, como os outros nitratos, a nitroglicerina converte o óxido nítrico (NO) no corpo humano e posteriormente vai ativar uma 
cascata de mediadores químicos e vai aumentar a recaptação no retículo sarcoplasmático do cálcio, abrindo os canais de potássio bloqueado pelo cálcio que foi absorvido. Segundo Kim K. H. et. al. (2021), sua indicação aprovada pela FDA é para alívio agudo do ataque cardíaco, doença arterial coronariana e angina de peito. $[51,52]$

Por fim, uma das doenças mais tratadas com uso off-label de medicamentos é a arritmia que é caracterizada como um distúrbio no ritmo cardíaco, podendo ter como causa lesão do tecido e/ou irregularidade da condução elétrica. Essa alteração pode ser diagnosticada como bradiarritmia ou taquiarritmia dependendo da frequência cardíaca apresentada ambos fora da faixa de referência; quando não monitorados podem resultar no desenvolvimento ou agravamento de cardiopatias em pacientes pediátricos.[41] Segundo estudo realizado na Inglaterra durante 20 anos, a incidência de arritmias foi de 24,4 para cada 100 mil nascidos vivos, sendo as arritmias mais comuns a Taquicardia por Reentrada Atrioventricular (TRAV), Bloqueio Atrioventricular (BAV) e o flutter atrial. [53, 54]

Um dos medicamentos utilizados para arritmia é a Amiodarona, seu mecanismo de ação consiste no tratamento de distúrbios graves do ritmo cardíaco, inclusive aqueles resistentes a outras terapias. O uso off-label neste caso, está relacionado à subdose, pois a dose de ataque regulamentada é $600 \mathrm{mg}$ a $1000 \mathrm{mg}$ para adultos, enquanto para crianças, é realizado um ajuste com administração de $10-20 \mathrm{mg} / \mathrm{kg} / \mathrm{dia}$, em 2 doses, no período de 7 a 10 dias. $[56,57]$

\section{CONCLUSÃO}

Diante do exposto, concluímos que o uso off-label se dá pelo uso de medicamentos fora do que são propostos e aprovados em suas bulas e por mais que seja regulamentado, ainda é uma grande dúvida na ciência e na sociedade. Mesmo com a larga utilização desta prática, existe a presença de riscos desconhecidos e um aumento de eventos adversos, podendo comprometer a segurança do paciente, visto que, a maior taxa de prescrição fora da bula é para crianças de 1 a 2 anos, afinal, possuem o sistema enzimático para metabolismo dos fármacos distinto ao dos adultos. Por outro lado, esse método é uma alternativa terapêutica quando há carência de tratamento para determinadas doenças e em casos emergenciais se torna um procedimento imediato, tendo em vista o processo para atualização da bula. Neste contexto, fica evidente a necessidade de pesquisas que priorizem o conhecimento farmacocinético e farmacodinâmico na população pediátrica, juntamente, reforçar as instruções ao profissional prescritor e aos profissionais de saúde, com a finalidade de racionalizar o tratamento e buscar evidência científica que suporte o uso offlabel, para que a experiência do paciente durante seu tratamento seja soberana a qualquer adversidade.

\section{RFERÊNCIAS BIBLIOGRÁFICAS}

[1]. Instituto Nacional de Cancêr (INCA) - Ministério da saúde,
Fases de desenvolvimento de um novo medicamento, 2021 Jul 23. Available from: https://www.inca.gov.br/pesquisa/ensaios-clinicos/ fases-desenvolvimento-um-novo-medicamento

[2]. Vasconselos D M M, Chaves G C, Azeredo T B, Silva R M. Política Nacional de Medicamentos em retrospectiva: um balanço de (quase) 20 anos de implementação. Ciência \& saúde coletiva Ago 2017.

[3]. Brazil - Presidência da República. Decreto $n^{\circ}$ 8.077, de 14 de agosto de 2013, do Ministério da Agricultura, Pecuária e Abastecimento. Regulamenta a Lei $n^{\circ} 6.360$, de 23 de setembro de 1976, que dispõe a Vigilância Sanitária a que ficam sujeitos os Medicamentos, as Drogas, os Insumos Farmacêuticos e Correlatos, Cosméticos, Saneantes e Outros Produtos, e dá outras Providências. Brasília: Diário Oficial da União; 2013. Available from: http://www.planalto.gov.br/ccivil_03/_Ato2011-2014/2013/ Decreto/D8077.htm\#art25

[4]. Anvisa.gov.br [internet] Brasil: Agencia de Vigilância Sanitária Registro de novos medicamentos: saiba o que é preciso. Available on http://www.anvisa.gov.br/

[5]. Aronson J K, Ferner E R. Unlicensed and off-label uses of medicines: definitions and clarification of terminology. British Pharmacological Society. 2017 August 05.

[6]. Nobre P F S. Prescrição Off-Label no Brasil e nos EUA: aspectos legais e paradoxos. Ciência \& Saúde Coletiva. 2013 May 3.

[7]. Paumgartten F J, Oliveira A C A X. Off label, compassionate and irrational use of medicines in Covid-19 pandemic, health consequences and ethical issues. Ciência \& Saúde Coletiva. 2020 september 9.

[8]. Diel J A C, Heineck I, Santos D B, Pizzol T S D. Off-label use of drugs by age in Brazilian children: a population study. Revista Brasileira de Epidemiologia. 2020 may 11.

[9]. Zen L, Marchetti F, Barbi E, Benini F. Off-label drugs use in pediatric palliative care. Italian Journal of Pediatric. 2018 November 29.

[10]. Gershon A, Shorter E. How amytal changed psychopharmacy: off-label uses of sodium amytal (1920-40). Sage Journals. 2019 May 30

[11]. Hans D. Katzberg, MD Ahmir H. Khan, MD Yuen T. So, MD, PhD. Assessment: Symptomatic treatment for muscle cramps (an evidence-based review). American Academy of Neurology. 2010

[12]. Pupo PP, Meira J O, Nasser J, Thomsen M. Considerações sobre sua terapêutica. Arquivos de Neuropsiquiatria. 2015 february 25.

[13]. Bodie A. Off-Label Use of Prescription Drugs. Congressional Research Service. 2021.

[14]. Tukayo B L A, Sunderland B, Parsons R, Czarniak P. High prevalence of off-label and unlicensed paediatric prescribing in a hospital in Indonesia during the period Aug. - Oct. 2014. Plos One. 2020 January 14.

[15]. Cabral H B, Silvestre G F, Neto A G. As Relações Jurídicas e a Pandemia da COVID-19. 1st ed. Campos dos Goytacazes, RJ Brazil. 2020. 
[16]. Reis O G C, Acesso a Antineoplásicos na Saúde Suplementar No Estado De São Paulo: concepções de prescritores e gestores de operadoras de saúde. Fundação Getúlio Vargas: Escola D Administração De Empresas de São Paulo. 2020

[17]. Bonati M, Aigrain E J, Choonara I. Licensed medicines, off-label use or evidence-based. Which is most important?. BMJ Journals Archives of Diseases in Childhood. 2016 August 17

[18]. Unlicensed and "off-label" Medicines Information for patients, parents and carers. Oxford University Hospitals. 2014 July. (16)

[19]. Duarte L S M, Torres L V, Coutinho M B, Wanderley T L $R$, Macedo $C L$. Informes e cuidados no uso de medicamentos offlabel frente à pacientes pediátricos. Residência Multiprofissional em Saúde da Criança - REMUSC - Complexo de Pediatria Arlinda Marques. 2018

[20]. Conselho Federal de Medicina (CFM - Brasil). Código de ética médica. Resolução №22 17 DE 27/ 0 9/2018.

[21]. Conselho Federal de Medicina (CFM - Brasil) ProcessoConsulta CFM No 2.437/2003 - PARECER CFM Nº 13/2004. Questiona se médicos possuem liberdade para prescrever medicamentos com indicações não aprovadas pela ANVISA. 2004 April 04.

[22]. Conselho Federal de Medicina (CFM - Brasil) PARECER CFM n ${ }^{\circ}$ 2/16. Prescrição de medicamentos off-label e Resolução CFM no 1.982/12. 2016 January 20

[23]. Silveira M C. O uso off-label de Medicamentos no Brasil. Fundação Oswaldo Cruz. 2019.

[24]. Nobre P F S. Off-label prescriptions in Brazil and in the US: legal aspects and paradoxes. Revista da associação brasileira de saúde coletiva. 2011

[25]. Barbosa C. Prescrição off-label, direito à informação, consentimento informado e processo clinico eletrónico no direito português. Cadernos Ibero-Americanos de Direito Sanitário. 2016 July/September.

[26]. Tefera Y G, Gebresillassie B M, Befekadu T. Off-Label Drug Use in Hospitalized Children: A Prospective Observational Study at Gondar University Referral Hospital, Northwestern Ethiopia. The Official Journal of the International Society for Pharmacoeconomics and Outcomes Research. 2017

[27]. BARBOSA, C. et al. Prescrição off-label, direito à informação, consentimento informado e processo clinico eletrônico no direito português. Cad. Ibero-Amer. Dir. Sanit., Brasília, 5(3):157-179, jul./set, 2016.

[28]. Ferreira LA, Ibiapina C C, Machado M G P, Fagundes E D T. A alta prevalência de prescrições de medicamentos off-label e não licenciados em unidade de terapia intensiva pediátrica brasileira. Revista da Associação Médica Brasileira. 2012 february 1.

[29]. Pande M N R, Amarante P D C, Baptista T W F. Este ilustre desconhecido: considerações sobre a prescrição de psicofármacos na primeira infância. Ciência \& Saúde Coletiva. 2020 June 6.

[30]. Koszma E I A, Bispo A J B, Santana I A O, Santos C N O B $D$. Uso de medicamentos off-label em unidade de terapia intensiva neonatal. Revista Paulista de Pediatria. 2021.
[31]. SANTOS, Armenio Aguiar; LIMA, José Milton de Castro; "Fisiologia Hepática",. In: Sistema Digestório: Integração BásicoClínica. São Paulo: Blucher, 2016. p. 575 -602

[32]. Selig F A. Panorama e Estratégias no Diagnóstico e Tratamento de Cardiopatias Congênitas no Brasil. Arquivos Brasileiros de Cardiologia. 2020 Dec 06

[33]. Pfizer [Internet] os diferentes tipos de cardiopatias; c2019 [cited 2021 july 21]. Available from https://www.pfizer.com.br/ noticias/ultimas-noticias/os-diferentes-tipos-de-cardiopatia

[34]. Boletim da Sociedade de Pediatria de São Paulo Cardiopatias Congênitas https://www.spsp.org.br/site/asp/boletins/ AtualizeA5N6.pdf

[35]. Brandão C. Abreu S. Pinto F. A doença cardiovascular em idade pediátrica. Orientações de Prática Clínica e Referenciação. Centro de Referência de Cardiopatias Congénitas do CHULC. 2019 september.

[36]. Gonçalves M G, Heineck I. Frequência de prescrições de medicamentos off-label e não licenciados para pediatria na atenção primária à saúde em município do sul do Brasil. Sociedade de pediatria de São Paulo. 2015.

[37]. Costa H T, Costa T X, Oliveira G O. Use of off-label and unlicensed medicines in neonatal intensive care. PLOS One. 2018

[38]. Lima A S. Dimensionamento do uso off-label de medicamentos, 2016. Available from: https://oswaldocruz.br/ revista_academica/content/pdf/Edicao_12_Lima_Adrienne_ Simeoni.pdf

[39]. Comité de Medicamentos de la Asociación Española de Pediatría. Pediamécum. Edición 2015. ISSN 2531-2464. Disponível em https://www.aeped.es/category/pediamecum/ cardiologia?page $=6$

[40]. Primacor [bula] 6, Boulevard de l'Europe 21800, Quetigny - França. Delpharm Dijon

[41]. Rodrigues, M., de Oliveira, J. C., Carvalho, F., Silva, H., Moreira, C., Granja, S., \& Gonçalves, A. Kawasaki disease and cardiovascular complications in pediatrics. Nascer e crescer - Birth and growth medical journal. 2018

[42]. Moreira H S S. Avaliação dos preditores de risco para doença de Kawasaki complicada numa população não japonesa. Mestrado integrado em medicina U. Porto

[43]. Gonçalves R C B, Silva S D. Doença de kawasaki: a importância do seu reconhecimento precoce. 2019

[44]. Moreira H S S. Avaliação dos preditores de risco para doença de Kawasaki complicada numa população não japonesa, 2020. Available from: https://repositorio-aberto.up.pt/ bitstream/10216/128404/2/411827.pdf

[45]. Sociedade Brasileira de Pediatria, Departamento Científico de Reumatologia (2019-2021), №6, Doença de Kawasaky, 2019 Nov .Available from: https://www.sbp.com.br/fileadmin/user_ upload/_22196c-DocCient_-_Doenca_de_Kawasaki.pdf

[46]. Marchesi, A., Tarissi de Jacobis, I., Rigante, D. et al. Kawasaki disease: guidelines of Italian Society of Pediatrics, part II - treatment of resistant forms and cardiovascular complications, follow-up, lifestyle and prevention of cardiovascular risks. Ital J Pediatr 44, 103 (2018). https://doi.org/10.1186/s13052-018-0529-2 
[47]. Comitê Coordenador da Diretriz de Insuficiência Cardíaca. Diretriz Brasileira de Insuficiência Cardíaca Crônica e Aguda. Arq Bras Cardiol. 2018; 111(3):436-539

[48]. Madriago E, Silberbach M. Heart Failure in Infants and Children. Pediatrics in review. 2010

[49]. Brokmeier H, Kido K. Off-label Use for Direct Oral Anticoagulants: Valvular Atrial Fibrillation, Heart Failure, Left Ventricular Thrombus, Superficial Vein Thrombosis, Pulmonary Hypertension-a Systematic Review. Annals of Pharmacotherapy. 2021;55(8):995-1009. doi:10.1177/1060028020970618

[50]. Pedraza LL, Machado BB, Souza RH, Silva ERR. Desenvolvimento e teste de um software para monitoramento à distância de pacientes com insuficiência cardíaca por short message service. Proceedings of $38^{a}$ Semana Científica do hospital de clínicas de Porto Alegre, 2018 Out 22 - 26, Porto Alegre, Brasil. $p 15$.

[51]. Kim KH, Kerndt CC, Adnan G, et al. Nitroglycerin. [Updated 2021 Aug 7]. In: StatPearls [Internet]. Treasure Island (FL): StatPearls Publishing; 2021 Jan-. Available from: https:// www.ncbi.nlm.nih.gov/books/NBK482382/?report=classic

[52]. Ho-Leung Fung Annual Review of Pharmacology and Toxicology 2004 44:1, 67-85

[53]. Magalhães LP, Guimarães ICB, Melo SL, Mateo EIP, Andalaft RB, Xavier LFR, et al. Diretriz de Arritmias Cardíacas em Crianças e Cardiopatias Congênitas SOBRAC e DCC - CP. Arq Bras Cardiol 2016; 107(1Supl.3):1-58

[54]. Szpalher A S, Batalha M C. Arritmias cardíacas: Diagnósticos de Enfermagem baseadosna Taxonomia da NANDA-I (2018-2020). Revista Eletrônica Acervo Saúde. 2019

[55]. Comité de Medicamentos de la Asociación Española de Pediatría. Pediamécum. Edición 2015. ISSN 2531-2464. . Disponivel em: https://www.aeped.es/comite-medicamentos/ pediamecum/amiodarona. Consultado el 29/09/2021.

[56]. Cloridrato de Amiodarona [Bula] Rua Conde Domingos Papaiz, 413 - Suzano - SP. Sanofi Medley Farmacêutica.

[57]. Cavalcante T. o uso crônico da amiodarona, seu manejo clínico e avaliação de protocolos de utilização. Universidade de Brasília, residência multiprofissional em farmácia clínica, especialidade cardiopulmonar. 2021 